\title{
Intramedullary Metastasis of Medulloblastoma: A Case Report
}

\author{
Abderrazzak Bertal1 ${ }^{1}$, Alberic Fabrice Bocco ${ }^{*}(\mathbb{D}$, Ismaïl Mahazou1, Asmaa Harrach², \\ Nihad da Silva ${ }^{3}$, Mohamed Yassine Haouas ${ }^{1}$, Khadija Ibahioin ${ }^{1,4}$, Said Hilmani' ${ }^{1}$, Mehdi Karkouri3 \\ Abdelhakim Lakhdar ${ }^{1,4}$ \\ ${ }^{1}$ Department of Neurosurgery, Ibn Rochd University Hospital Center, Casablanca, Morocco \\ ${ }^{2}$ Department of Hematology and Pediatric Oncology, Ibn Rochd University Hospital Center, Casablanca, Morocco \\ ${ }^{3}$ Department of Pathology, Ibn Rochd University Hospital Center, Casablanca, Morocco \\ ${ }^{4}$ Laboratory of Research on Neurologic and Neurosensorial Diseases and Handicap, Faculty of Medicine, Hassan II University, \\ Casablanca, Morocco \\ Email: ^fabricebocco@gmail.com
}

How to cite this paper: Bertal, A., Bocco, A.F., Mahazou, I., Harrach, A., da Silva, N., Haouas, M.Y., Ibahioin, K., Hilmani, S., Karkouri, M. and Lakhdar, A. (2021) Intramedullary Metastasis of Medulloblastoma: A Case Report. Open Journal of Modern Neurosurgery, 11, 90-96. https://doi.org/10.4236/ojmn.2021.112010

Received: November 9, 2020

Accepted: April 10, 2021

Published: April 13, 2021

Copyright $\odot 2021$ by author(s) and Scientific Research Publishing Inc. This work is licensed under the Creative Commons Attribution International License (CC BY 4.0).

http://creativecommons.org/licenses/by/4.0/ (c) (i) Open Access

\begin{abstract}
Medulloblastoma $(\mathrm{MB})$ is a malignant brain tumor with a usual potential for leptomeningeal spread. Intramedullary metastases of $\mathrm{MB}$ are rare and there are very few cases reported in the literature. Here, we report the case of an 18 -year-old man with intramedullary spinal cord metastasis of MB occurring 9 years after the first diagnosis. The patient presented a 2-month history of progressive weakness in both lower limbs associated with urinary incontinence. Magnetic resonance imaging (MRI) demonstrated a large intramedullary spinal cord tumor extending from $\mathrm{T} 10$ to L1. The patient underwent surgical decompression and adjuvant therapy. Histological examination confirmed the diagnosis of classic MB metastasis. Postoperatively, the neurological status was stationary. Intramedullary metastasis of medulloblastoma is rare and difficult to manage with a poor prognosis. Comprehensive studies on the medulloblastoma dissemination mechanisms and clinical trials are needed to assess combined therapeutic approaches on metastases of MB.
\end{abstract}

\section{Keywords}

Medulloblastoma, Intramedullary Metastasis, Classic, Spinal Cord

\section{Introduction}

Medulloblastoma (MB) is the most common malignant tumor of childhood and constitutes approximately $30 \%$ of pediatric brain tumors [1]. MB starts com- 
monly in the posterior fossa and tends to disseminate through the cerebrospinal fluid pathways. The conventional treatment is multimodal therapy including maximum safe resection of the tumor, followed by craniospinal radiation with or without chemotherapy. Adjuvant treatment is often considered to avoid the dissemination of a local tumor and to control the disease. The posterior fossa and leptomeningeal area are the most common recurrence location in the central nervous system. Intramedullary spinal cord metastasis is rare and to date, there are few cases reported in the literature [2] [3] [4] [5] [6]. We reported a case of intramedullary spinal cord metastasis of $\mathrm{MB}$ which occurred 9 years after first management of the vermian medulloblastoma without a local recurrence. This case report also aims to discuss the pathogenesis of ISCM in MB and the role of surgery for managing medulloblastoma recurrence according to the literature.

\section{Case Report}

An 18-year-old man was admitted for 2-month progressive weakness in both lower limbs associated with urinary incontinence. The patient had undergone total resection of a classic vermian medulloblastoma 9 years ago (World Health Organization, grade IV). The spinal cord magnetic resonance imaging (MRI) and CSF cytology were negative. Postoperative craniospinal irradiation was performed with $36 \mathrm{~Gy}$ on the craniospinal axis and boost of $54 \mathrm{~Gy}$ on the posterior fossa to the tumor bed. The patient received also chemotherapy combining vincristine, cisplatin and cyclophosphamide. The patient had remained asymptomatic during the entire follow-up until the present admission. On clinical examination, muscle strength was assessed as $2 / 5$.

Spinal MRI revealed a large intramedullary tumor lesion extending from T10 to L1 with a syrinx above and below the mass. The tumor lesion appears iso-intense on the $\mathrm{T} 1$ and $\mathrm{T} 2$-weighted images with enlargement of the spinal cord by the tumor. There was also another intramedullary nodular lesion at T2 - T3. Both tumor lesions took on the contrast medium after administration of gadolinium. There were also small stepped enhancements to the thoracolumbar leptomeningeal space [Figure 1]. The brain MRI did not show any sign of local recurrence in the surgical bed of the posterior cerebral fossa [Figure 2]. Based on the patient's history, medulloblastoma recurrence was suspected.

The patient underwent laminectomy and excision of the lesion at T10 to L1. Intraoperatively, there was no cleavable plane between the tumor and the spinal cord. Partial resection of the lesion was performed to achieve decompression of the effect of tumor mass on the spinal cord. The histopathological examination revealed a malignant tumor proliferation compatible with a metastasis of classic medulloblastoma. The tumor cells appeared with dense, hyperchromatic, crushed, agglutinated nuclei with scanty cytoplasm [Figure 3]. Postoperatively, neurological status was stationary and complementary chemotherapy was administered to the patient. Unfortunately, the patient died four months later for disseminated disease. 


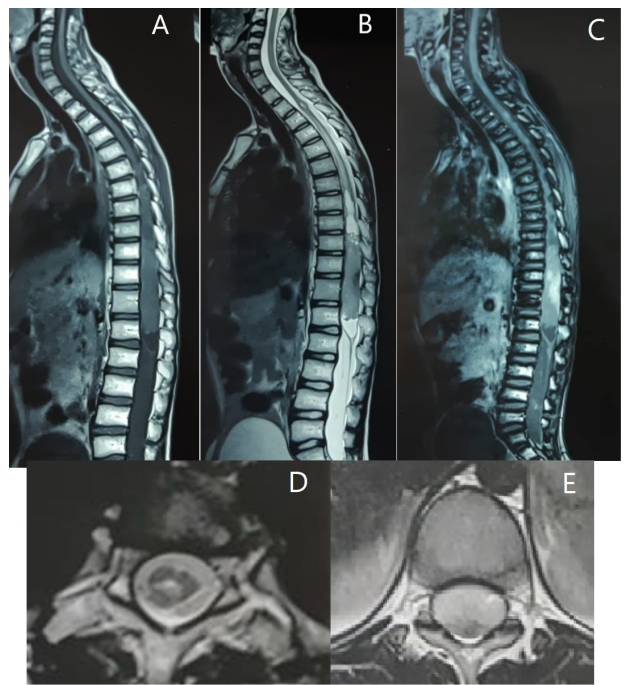

Figure 1. Spinal magnetic resonance imaging (MRI). Sagittal (A) T2-weighted and (B) T1-weighted images showing an intramedullary lesion from T10 to L1 with a syrinx and another intramedullary nodular lesion at T2 - T3; (C) Sagittal gadolinium image showing heterogenously contrast enhancement of both lesion and small stepped enhancements to the thoracolumbar leptomeningeal space; (D) Axial T2-weighted image at T2 - T3; (E) Axial T2-weighted image at the T10 showing enlargement of the thoracic spinal cord.

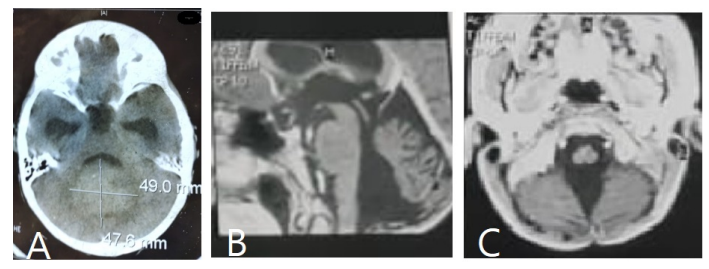

Figure 2. (A) Brain CT scan at initial diagnosis showing a posterior fossa tumor. Brain magnetic resonance imaging (MRI): (B) Sagittal T1-weighted image and (C) Axial T1-weighted image after gadolinium administration showing no sign of local recurrence in the posterior fossa.
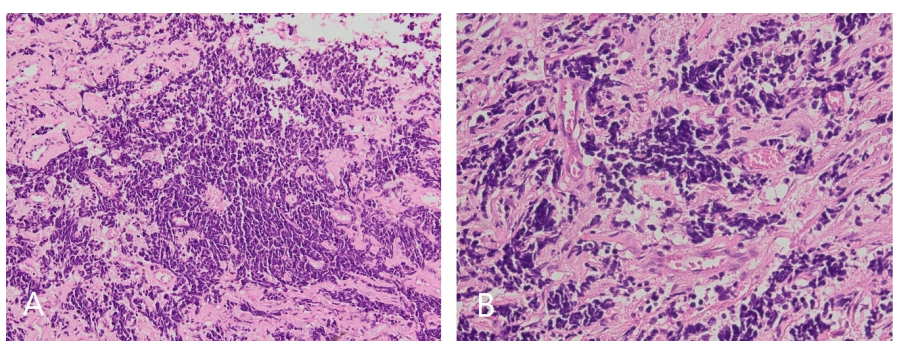

Figure 3. Microscopic examination of the surgical specimen from the spinal lesion revealing a medulloblastoma. (A) Hematoxylin and eosin staining, magnification $\times 200$ : A malignant diffuse tumor proliferation; (B) Hematoxylin and eosin staining, magnification $\times 400$ : Details of tumor proliferation. The cells had hyperchromatic, crushed, agglutinated nuclei with scanty cytoplasm.

\section{Discussion}

The 2016 classification of the World Health Organization (WHO) on brain tumors defines $\mathrm{MB}$ as a separate entity from tumors, and equally defines $\mathrm{MB}$ ac- 
cording to its molecular and histopathological characteristics [7]. Histologically, $\mathrm{MB}$ is classified into the following subtypes: classic, desmoplastic/nodular, large cell/anaplastic, and MB extensive nodularity. Furthermore, four molecular subgroups of $\mathrm{MB}$ are described namely: activated wingless (WNT), activated sonic hedgehog (SHH), group 3 and group 4. Each subgroup has gene expression profiles, an age of onset and a distinct prognosis [8] [9].

The revised WHO classification allows for an integrated diagnosis of $\mathrm{BM}$ which helps to predict the aggressive nature of the tumor and therefore the prognosis. The goal is also therapeutic in order to define according to each group a protocol to guide the management.

Medulloblastoma (MB) is a high-grade tumor that frequently resides in the cerebellum in the posterior fossa and has a high potential for spreading on the craniospinal axis. Different mechanisms are described to explain seeding to other parts of the brain and spinal cord. Common theory suggests that tumor cells invade CSF by disrupting the blood-brain barrier during tumor progression due to the proximity of the 4th ventricle to the vermis. Surgery can also allow iatrogenic contamination of the subarachnoid spaces by tumor cells. Moreover, other authors have suggested the hematogenous propagation of tumor cells [10], explaining the extraneural metastatic locations of medulloblastoma. Dissemination of $\mathrm{MB}$ frequently occurs through the leptomeningeal space, but the intramedullary site is relatively poorly reported and would be explained by the passage of tumor cells through the central duct. Genetic factors might allow medulloblastoma tumor cells to grow in the brain and spinal cord. Jenkins et al. [11] described two genes expressed in MB that would promote the dissemination of medulloblastoma tumor cells: the nuclear translocator of the aryl hydrocarbon receptor (Arnt) and the dissociation inhibitor of GDP 2 (Gdi2). Arnt and Gdi2 are believed to increase cell motility and invasiveness, which causes cells to detach from the primary tumor mass.

CSF cytology and craniospinal MRI are recommended to assess the spread of MB to the neuraxis [12]. These explorations are useful in order to be able to stratify the medulloblastoma and thus define the management strategy according to the "standard risk" and "high risk" group. Advances in treatment over the past three decades have improved the survival of patients with medulloblastoma [8]. Conventional treatment includes surgery along with radiotherapy and/or chemotherapy. The goal of adjuvant therapy is to limit the progression of tumor disease or the dissemination. Koschmann et al. [13] reported in a case series that the median time between diagnosis and tumor recurrence was 18 months (range, 3.6 to 62.6). The interval between initial diagnosis and metastasis of $\mathrm{MB}$ in our case was approximately 9 years.

Intramedullary metastases from medulloblastoma have been poorly reported in the literature [2] [3] [4] [5] [6]. To our knowledge, only 7 cases have been published. The incidence is underestimated because intramedullary metastases are generally not clearly identified with leptomeningeal metastases. Current treatment strategies for relapsed BM include maximum safe resection combined 
with chemotherapy and/or re-irradiation [11]. Surgical treatment of intramedullary metastases allows the histological and molecular diagnosis, but its benefits in improving neurological status, quality of life and survival remains a controversial matter. In our case, the surgical treatment did not influence the postoperative neurological status. In contrast, Goyal et al. [2] reported the case of a 14 -year-old child who underwent surgery for intramedullary metastasis. The patient improved neurologically initially post-operatively before dying from the spread of the disease. Inoue et al. [3] also described intramedullary metastasis at initial diagnosis in a 4-year-old boy who was managed with biopsy and chemotherapy. The child died about three months later from the spread of the disease. Madhugiri et al. [4] also reported a case of spinal cord metastasis diagnosed in a 19-year-old patient treated with radiotherapy and chemotherapy with an unchanged neurological status and who was a loss to follow-up.

Surgery is an option of treatment, but it does not control the spread of the disease. Re-irradiation with stereotactic radiosurgery appeared to have no potential toxicity if recommended dose constraints to the spinal cord are carefully observed [14]. Distant failure appears to be the rule despite re-irradiation of recurrent medulloblastoma. However, retreatment achieves potential benefits for the survival with the association between longer survival with chemotherapy and additional radiotherapy [13]. The median survival after relapse was 10.3 months (range, 1.3 to 80.5) [13]. Clinical trials are needed to assess combined therapeutic approaches on metastases of MB.

\section{Conclusion}

The follow-up of patients with MB is crucial to diagnose recurrence early. The metastases of $\mathrm{MB}$ and especially of intramedullary spinal metastases are difficult to manage with a poor prognosis. Knowledge of the medulloblastoma dissemination mechanisms seems to be fundamental and could help define a more effective therapeutic strategy.

\section{Acknowledgements}

The authors thank Eric Akpoviroro Obar for his support in arranging the paper and Said Wati for data provision.

\section{Patient Consent}

The patient consent was obtained for this article from the guardian.

\section{Authors Contributions}

Authors' contribution provides literature review, manuscript preparation and editing.

\section{Conflicts of Interest}

The authors declare that there are no relevant conflicts of interest. 


\section{References}

[1] Khanna, V., Achey, R.L., Ostrom, Q.T., Block-Beach, H., Kruchko, C., Barnholtz-Sloan, J.S. and de Blank, P.M. (2017) Incidence and Survival Trends for Medulloblastomas in the United States from 2001 to 2013. Journal of Neuro-Oncology, 135, 433-441. https://doi.org/10.1007/s11060-017-2594-6

[2] Goyal, A., Cajigas, I., Ibrahim, G.M., Brathwaite, C.D., Khatib, Z., Niazi, T., Bhatia, S. and Ragheb, J. (2018) Surgical Treatment of Intramedullary Spinal Metastasis in Medulloblastoma: Case Report and Review of the Literature. World Neurosurgery, 118, 42-46. https://doi.org/10.1016/j.wneu.2018.06.250

[3] Inoue, T., Kumabe, T., Takahashi, T., Nakajima, T., Watanabe, M. and Tominaga, T. (2007) Spinal Intramedullary Metastasis of Medulloblastoma at Initial Diagnosis. Child s Nervous System, 23, 113-116. https://doi.org/10.1007/s00381-006-0167-7

[4] Madhugiri, V.S., Pandey, P., Indira Devi, B., Santosh, V. and Yasha, T.C. (2012) Intramedullary Metastasis in a Case of Vermian Medulloblastoma. British Journal of Neurosurgery, 26, 278-280. https://doi.org/10.3109/02688697.2011.603855

[5] Barnwell, S.L. and Edwards, M.S.B. (1986) Spinal Intramedullary Spread of Medulloblastoma: Case Report. Journal of Neurosurgery, 65, 253-255. https://doi.org/10.3171/jns.1986.65.2.0253

[6] Zumpano, B.J. (1978) Spinal Intramedullary Metastatic Medulloblastoma: Case Report. Journal of Neurosurgery, 48, 632-635. https://doi.org/10.3171/jns.1978.48.4.0632

[7] Louis, D.N., Perry, A., Reifenberger, G., von Deimling, A., Figarella-Branger, D., Cavenee, W.K., Ohgaki, H., Wiestler, O.D., Kleihues, P. and Ellison, D.W. (2016) The 2016 World Health Organization Classification of Tumors of the Central Nervous System: A Summary. Acta Neuropathologica, 131, 803-820. https://doi.org/10.1007/s00401-016-1545-1

[8] von Bueren, A.O., Kortmann, R.-D., von Hoff, K., Friedrich, C., Mynarek, M., Müller, K., Goschzik, T., zur Mühlen, A., Gerber, N., Warmuth-Metz, M., Soerensen, N., Deinlein, F., Benesch, M., Zwiener, I., Kwiecien, R., Faldum, A., Bode, U., Fleischhack, G., Hovestadt, V., Kool, M., Jones, D., Northcott, P., Kuehl, J., Pfister, S., Pietsch, T. and Rutkowski, S. (2016) Treatment of Children and Adolescents with Metastatic Medulloblastoma and Prognostic Relevance of Clinical and Biologic Parameters. Journal of Clinical Oncology, 34, 4151-4160. https://doi.org/10.1200/JCO.2016.67.2428

[9] Kijima, N. and Kanemura, Y. (2016) Molecular Classification of Medulloblastoma. Neurologia Medico-Chirurgica, 56, 687-697. https://doi.org/10.2176/nmc.ra.2016-0016

[10] Garzia, L., Kijima, N., Morrissy, A.S., De Antonellis, P., Guerreiro-Stucklin, A., Holgado, B.L., Wu, X., Wang, X., Parsons, M., Zayne, K., Manno, A., Kuzan-Fischer, C., Nor, C., Donovan, L.K., Liu, J., Qin, L., Garancher, A., Liu, K.-W., Mansouri, S., Luu, B., Thompson, Y.Y., Ramaswamy, V., Peacock, J., Farooq, H., Skowron, P., Shih, D.J.H., Li, A., Ensan, S., Robbins, C.S., Cybulsky, M., Mitra, S., Ma, Y., Moore, R., Mungall, A., Cho, Y.J., Weiss, W.A., Chan, J.A., Hawkins, C.E., Massimino, M., Jabado, N., Zapotocky, M., Sumerauer, D., Bouffet, E., Dirks, P., Tabori, U., Sorensen, P.H.B., Brastianos, P.K., Aldape, K., Jones, S.J.M., Marra, M.A., Woodgett, J.R., Wechsler-Reya, R.J., Fults, D.W. and Taylor, M.D. (2018) A Hematogenous Route for Medulloblastoma Leptomeningeal Metastases. Cell, 172, 1050-1062.e14. https://doi.org/10.1016/j.cell.2018.01.038

[11] Jenkins, N.C., Kalra, R.R., Dubuc, A., Sivakumar, W., Pedone, C.A., Wu, X., Taylor, 
M.D. and Fults, D.W. (2014) Genetic Drivers of Metastatic Dissemination in Sonic Hedgehog Medulloblastoma. Acta Neuropathologica Communications, 2, Article No. 85. https://doi.org/10.1186/s40478-014-0085-y

[12] Fouladi, M., Gajjar, A., Boyett, J.M., Walter, A.W., Thompson, S.J., Merchant, T.E., Jenkins, J.J., Langston, J.W., Liu, A., Kun, L.E. and Heideman, R.L. (1999) Comparison of CSF Cytology and Spinal Magnetic Resonance Imaging in the Detection of Leptomeningeal Disease in Pediatric Medulloblastoma or Primitive Neuroectodermal Tumor. Journal of Clinical Oncology, 17, 3234-3237.

https://doi.org/10.1200/JCO.1999.17.10.3234

[13] Koschmann, C., Bloom, K., Upadhyaya, S., Geyer, J.R. and Leary, S.E.S. (2016) Survival after Relapse of Medulloblastoma. Journal of Pediatric Hematology/ Oncology, 38, 269-273. https://doi.org/10.1097/MPH.0000000000000547

[14] Boyce-Fappiano, D., Elibe, E., Zhao, B., Siddiqui, M.S., Lee, I., Rock, J., Ryu, S. and Siddiqui, F. (2017) Reirradiation of the Spine with Stereotactic Radiosurgery: Efficacy and Toxicity. Practical Radiation Oncology, 7, 409-417.

https://doi.org/10.1016/j.prro.2017.05.007 